\title{
Carnets
}

Revue électronique d'études françaises de l'APEF

Première Série - 2| 2010

L'équivoque

\section{Équivoque litteraire et contrat de lecture}

\section{Alain Trouvé}

\section{(2) OpenEdition \\ Journals}

Édition électronique

URL : http://journals.openedition.org/carnets/4447

DOI : $10.4000 /$ carnets.4447

ISSN : 1646-7698

Éditeur

APEF

Édition imprimée

Date de publication : 1 janvier 2010

Pagination : 6-17

Référence électronique

Alain Trouvé, «Équivoque litteraire et contrat de lecture », Carnets [En ligne], Première Série - 2 | 2010, mis en ligne le 16 juin 2018, consulté le 02 mai 2019. URL : http://journals.openedition.org/ carnets/4447 ; DOI : 10.4000/carnets.4447

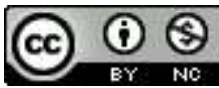

Carnets est mis à disposition selon les termes de la licence Creative Commons - Atribution - Pas d'utilisation commerciale 4.0 International. 


\title{
ÉQUIVOQUE LITTÉRAIRE ET CONTRAT DE LECTURE
}

ALAIN TROUVÉ

Université de Reims

alain.trouve@wanadoo.fr

\begin{abstract}
Résumé
L'équivoque (qui naît de son contraire, l'univoque) est d'abord affaire de genre et de contrat de lecture. Lorsque l'intention de l'auteur (nécessaire au contrat) devient peu perceptible, l'équivoque se décrit comme un protocole flottant. Elle concerne enfin l'interprétation : avec la complicité de l'auteur ou en prenant ses distances, le lecteur tend à réduire l'équivoque en univoque, à moins qu'il ne fasse parfois le contraire. Pour l'élaboration du sens, la fiction, notamment romanesque, est le terrain privilégié de l'équivoque.
\end{abstract}

\begin{abstract}
Equivocation, which derives from its opposite "univocity", is first of all a matter of genre and reading contract. When authorial intention, which is necessary to the contract, is blurred, equivocation can be described as a drifting course of action. Interpretation is also affected: with the author's complicity or by taking his distance with it, the reader aims to reduce equivocation into univocity, unless he is bent on doing exactly the opposite. As far as the construction of meaning is concerned, fiction, particularly novelistic fiction, is a choice nursing ground for equivocation.
\end{abstract}

Mots-clés: Contrat (pacte), protocole, genre, fiction, intention, interprétation, inconscient, intertexte. Keywords: contract (pact), protocol, genre, fiction, intention, interpretation, unconscious, intertext. 
S'il doit être fait un distinguo entre ambiguïté et équivoque, il réside dans la présence, au sein de l'équivoque, de l'étymon vox, vocis «voix, parole». L'ambiguïté et l'équivoque impliquent l'une et l'autre une hésitation, un balancement entre deux ou plusieurs interprétations. L'équivoque se rapporte plus nettement à un sujet, représenté par une parole. Toute parole est susceptible de nourrir l'équivoque, autrement dit les spéculations sur ce que veut dire l'émetteur. Plus complexe, souvent plus retorse, la parole littéraire s'ouvre selon une grande diversité de modalités à l'équivoque. Une parole est engagée dans un échange impliquant une réponse ; la parole littéraire, dont la réponse est différée, est affaire de contrat ou de pacte, ainsi que l'a souligné la critique à la fin du vingtième siècle dans le sillage de Philippe Lejeune. Pacte ostensible ou tacite, authentique ou trompeur, accepté ou subverti. La gamme des effets de genre est riche et variée, celle des effets de lecture ne l'est pas moins. Le partage du littéraire qui fonde l'esthétique moderne suppose en effet une part active et créatrice du lecteur (Rancière, 2007 et 2008). On s'efforcera donc de mettre en perspective quelques-uns des cas de figure les plus frappants touchant aux questions du genre, de l'intention et d'un partage lui-même incertain des rôles assumés par l'auteur et ses lecteurs.

\section{Postures auctoriales, genres et catégories esthétiques}

\section{Equivoque refusée}

Que l'équivoque ait un rapport sans doute privilégié avec le littéraire ne doit pas occulter le vaste champ des productions fondées sur son refus ou son rejet au second plan en tant que critère générique. Le continent fictionnel, du roman au conte, lui tourne le dos, avec la complicité du lecteur. Le fameux « il était une fois » est le sésame ouvrant sur l'autre monde de l'imaginaire, des rêves. Au fil des siècles et en dépit des crises qui l'ont parfois mis en péril, le genre romanesque a continué à vivre de cette alternative à la vie réelle proposée au lecteur, de cette virtualité des expériences affichée sans équivoque grâce à deux indices complémentaires et très fréquemment réunis : le sous-titre roman proposé dans le péritexte éditorial, avec la complicité de l'auteur, l'invention du nom de personnage qui signe véritablement l'entrée en fiction. La remarque sera toutefois à nuancer fortement en raison de la plasticité générique de cette forme narrative dont le seul critère stable est peut-être, comme on vient de le dire, de se donner comme fiction.

Dans le champ de l'écriture de soi, l'acte de naissance du genre autobiographique remonterait, suivant la démonstration de Philippe Lejeune (1975), à l'écriture des Confessions, en concordance avec l'émergence de l'individu dans les sociétés occidentales. Il est vrai que la première page pose avec une netteté remarquable un pacte qui se veut aussi pacte de vérité, et se fonde sur l'identité de l'Auteur, du Narrateur et du personnage (le 
Je narré). Symétriquement au pacte romanesque, le pacte autobiographique exclut à un premier niveau de lecture l'équivoque.

La distinction des genres paraît mieux accordée aux sociétés fondées sur un ordre hiérarchique pressenti comme immuable : monarchie absolue, suprématie d'un empereur ou d'une caste au pouvoir. La remarque vaut pour l'épopée, fruit de la société grecque ancienne dans sa version homérique ou célébration de la grandeur d'Auguste, chez Virgile. Au déploiement du genre correspond l'image d'une humanité célébrant les valeurs consensuelles : bravoure, service féodal ${ }^{1}$. II n'est pas sans intérêt de noter l'inflexion du roman moderne vers l'épopée à l'époque du réalisme socialiste, sous la plume d'un Nicolas Ostrovski (Et l'acier fut trempé) ou d'un Aragon (Les Communistes). L'époque ayant décidément changé, il ne s'agit que d'une inflexion.

L'âge classique fut comme on le sait celui de la théorisation des genres séparés, notamment au théâtre. La distinction entre tragédie et comédie qui fonctionne bien pour le théâtre de Racine prête déjà à discussion s'agissant de celui de Molière dont les commentateurs ont remarqué le fonds de noirceur sous-jacent, certains pour en féliciter l'auteur, d'autres pour le lui reprocher. Le Misanthrope ou Don Juan côtoient le tragique. II n'empêche : les procédés comiques sont en nombre suffisant pour disqualifier les mises en scène qui feraient l'impasse sur l'inscription générique dans le registre de la comédie. Don Juan peut bien être précipité en enfer dans une scène qui emprunte par ailleurs au merveilleux religieux, la pièce se termine sur les récriminations de Sganarelle réclamant ses gages.

\section{Equivoque assumée ou revendiquée, pactes flottants}

L'existence de catégories génériques non équivoques et identifiables grâce à une série de traits distinctifs ${ }^{2}$, fonde la possibilité d'équivoques d'écriture. On parlera alors d'équivoque revendiquée, dont les exemples sont tout aussi connus. Le drame romantique arbore le programme d'un mélange du comique et du tragique. Hugo qui tente de le théoriser dans la Préface de Cromwell en offre une version dans son théâtre, d'Hernani à Ruy Blas. On assiste ainsi à la naissance de nouvelles catégories esthétiques, fondées sur l'alliance de contraires et dont le caractère équivoque ne vaut en réalité que par l'attente de catégories plus pures. L'effet s'émousse donc très vite. De même, les demi-teintes, le clair-obscur propres à l'art baroque en littérature ou en peinture deviennent aisément identifiables comme indices d'une catégorie esthétique d'un autre ordre. Un certain amalgame du comique et du

\footnotetext{
${ }^{1}$ Voir à ce sujet les distinctions toujours valables à nos yeux posées par Lukàcs dans La Théorie du roman, entre l'univers épique, fondé sur "un système de valeurs achevé et clos » et l'univers romanesque dans lequel le héros est confronté à « l'altérité du monde extérieur ».

${ }^{2}$ Exploit, grandeur et démesure dans l'épopée qui rapproche le héros individuel et collectif de l'absolu divin.
} 
tragique est de la même façon la marque de fabrique parfaitement identifiable du théâtre shakespearien, précurseur à bien des égards du drame romantique.

Que l'équivoque se fabrique à partir de l'univoque se démontrerait encore en observant le conte fantastique dans la seconde moitié du $19^{\text {ème }}$ siècle. Selon les deux logiques mises en concurrence dans le Horla le narrateur est un être en proie à une force surnaturelle maléfique ou simplement un fou. Tout l'art du conteur Maupassant consiste à faire partager à son lecteur l'hésitation du héros en donnant à l'incroyable les apparences du vraisemblable, en mélangeant habilement le naturel et le surnaturel, les codes du récit réaliste et ceux du conte. Hoffmann et Poe agissent de même dans leurs cultures respectives. La disparition de cette équivoque narrative, la résorption du fantastique au vingtième siècle dans des formes plus ouvertement apparentées au fantasme chez un auteur comme Lovecraft s'explique sans doute par l'évolution des croyances dans le lectorat plus accoutumé à la vulgarisation de la pensée scientifique.

A l'intérieur du champ romanesque dont la plasticité accueille divers sous-genres on observe notamment au vingtième siècle une tendance à jouer sur des formes déceptives : nombre de récits sont ainsi construits comme le roman policier sur le modèle de l'enquête avec mystère, indices, détective plus ou moins déclaré. Gide, Faulkner, Queneau, Modiano, parmi bien d'autres jouent avec les codes du roman policier de manière temporaire. L'enquête cède en effet la place selon des modalités propres à chaque auteur à une autre recherche : expression par le comique anachronique et les jeux de mots de l'absurdité du monde contemporain chez Queneau (Les Fleurs bleues), quête identitaire chez le sujet écrivant et lisant chez Modiano (La ronde de nuit), par exemple. Le pacte de lecture flottant est une invite à lire au-delà de l'histoire racontée, il ouvre le champ interprétatif.

\section{La mystification et le pacte impossible}

Terminons par deux cas extrêmes. La mystification littéraire propose un faux pacte générique. A l'univoque peut se substituer la duplicité, dès lors qu'est reconnue la mystification par le lecteur. Ainsi le Traité du style d'Aragon pastiche les traités de rhétorique pour mieux s'en éloigner, à moins que le livre ne soit finalement reçu pour ce qu'il est sans doute, un vrai faux traité. Les vérités en trompe-l'œil sont aussi à identifier à l'intérieur du volume qui insère en collage des coupures de journaux mettant en cause pour vol avec effraction un certain Louis Aragon, homonyme de l'auteur. II faut alors, pour démonter la supercherie, recourir à l'enquête externe. De même le volume Pléiade consacré à SaintJohn Perse et réalisé sous le contrôle de l'auteur débute par une notice biographique dans le plus pur style scientifique de la collection. Mais elle est truffée d'affabulations peu évidentes à détecter par un lecteur non averti qui ne lirait pas le volume in extenso pour trouver les passages éventant la supercherie ou ne s'aiderait pas, là encore, des commentaires des 
spécialistes $^{3}$. La mise au jour de l'équivoque par mystification implique l'intervention d'une communauté de lecteurs.

L'autre cas limite est celui de l'autofiction. On connaît la fortune de cette expression inventée par Serge Doubrovsky à propos de son livre Fils pour donner un nom à une forme nouvelle d'écriture de soi. Récusant l'incompatibilité supposée entre les pactes autobiographique et romanesque, l'auteur prétend s'y raconter à travers des histoires inventées. Le je narrant et le je narré renvoient à la personne de Serge Doubrovsky, ce qui lui arrive, non. L'antinomie pourrait disparaître si l'on s'avisait de recourir ici avec Ricœur (1990) à la distinction entre une identité de personne (le sujet au sens grammatical des actions successivement narrées), et l'identité de caractère (les traits révélés par les anecdotes). On verrait ainsi dans l'autofiction un essai de renouvellement de l'écriture de soi accompli par un auteur féru de psychanalyse et familier des ruses de l'inconscient, grand pourvoyeur d'affabulations. Même fictionnelle, l'écriture de Doubrovsky continue par son préfixe « auto » à renvoyer à un objet privilégié : la personne de l'auteur.

II n'en va pas de même, à notre sens de ce qu'on pourrait appeler la nébuleuse autofictionnelle, qui signale simplement des flottements de contrat à l'intérieur d'une écriture et fait l'objet de divers malentendus. J'y reviendrai.

L'équivoque n'est donc qu'une des formes du pacte de lecture littéraire, adossé à la notion de genre, elle-même historiquement déterminée. Sa reconnaissance est un acte individuel ou collectif, requérant la communauté des interprètes. Situer un texte parmi des références génériques engage déjà l'interprétation et pose la question de l'intention ayant présidé à l'écriture de ce texte, sans doute aussi de la maîtrise du sujet auteur sur son texte et sur le sens.

\section{Intention et sens littéraire}

II n'est pas sûr que la notion de pacte s'applique à tout le champ de la production littéraire. Ce problème est lié à celui de l'intention littéraire ${ }^{4}$.

\section{Difficultés de l'univocité}

Reprenons le cas d'école des Confessions. «Je forme une entreprise qui n'eut jamais d'exemple [...]. Je veux montrer à mes semblables un homme dans toute la vérité de la nature ; et cet homme ce sera moi », déclare Jean-Jacques au seuil de son livre. Le pacte autobiographique est un pacte de vérité sur soi, de lucidité extrême qui apparenterait son auteur à Dieu. II impliquerait qu'un individu puisse avoir accès à l'intégralité de son

\footnotetext{
${ }^{3}$ Pour ces deux cas de figure, voir notre article « Mystification et jeu littéraire ».

${ }^{4}$ Intention déclinée en trois termes par Umberto Eco : intentio scriptoris, intentio operis, intentio lectoris (Les limites de l'interprétation).
} 
psychisme. La notion d'inconscient perdrait toute pertinence pour un tel sujet. II n'en est rien, heureusement sans doute. L'écriture littéraire de l'autobiographie dépasse l'intention auctoriale et offre au lecteur un espace d'interprétation qui réintroduit l'équivoque au sein de l'univoque. Lejeune soumet le texte de Rousseau à une écoute analytique et montre à l'œuvre un désir inconscient qui ne se dit pas directement et emprunte failles et détours pour se donner à lire. Starobinski, dans son essai La transparence et l'obstacle, suggère de même que la volonté de clarté se heurte à la matérialité de l'écriture et à la polysémie résultant d'un usage littéraire du signe.

Si l'on élargit le propos, la parole divine, détentrice de la Vérité serait en son essence incompatible avec l'équivoque, ce qui pose au passage la question du statut de certains textes à visée apologétique comme Les Pensées. Les querelles entre exégètes des différentes religions montrent que les fidèles, confrontés ne serait-ce qu'à la lettre d'un texte, sont loin de s'entendre. Chacun se situe pourtant dans le déni d'équivoque, s'attribuant l'orthodoxie et renvoyant à l'autre l'hérésie, en dépit ou en raison d'une posture commune d'allégeance à la Vérité divine. L'humanité en a payé le prix fort. Le déni d'équivoque flirte avec la terreur et fait mauvais ménage avec le littéraire. A l'opposé de ces querelles entre experts en vérité se situerait la lecture facétieuse et irrévérencieuse qui amène Joël Martin (2009), orfèvre en la matière, à débusquer des contrepèteries dans le texte biblique (« Le passage de la mer Rouge »). De ce petit exemple on peut tirer deux remarques. Le statut d'un texte n'est pas entièrement décidable dans une perspective essentialiste et pourrait bien dépendre de l'attitude adoptée par les lecteurs pour le lire. L'attitude littéraire est en rapport avec le feuilleté du signe arrimant un signifiant (matière sonore) à un signifié ; elle met aussi en jeu, pour le plaisir du lecteur, des significations sexuelles plus ou moins détournées, comme le montra en son temps Freud dans Le Mot d'esprit.

De l'univoque à l'équivoque

Le détournement de sens, licite ou non, réintroduit donc l'équivoque au sein de l'univoque. La perception de cette dimension de l'équivoque amène à distinguer un sens littéral et un sens interprété. La lecture immédiate des Confessions montre effectivement, dans le droit fil du pacte initialement proposé, un auteur avouant des actes plus ou moins honteux et pratiquant en quelque sorte comme les protestants la confession publique. L'analyse plus approfondie met au jour le plaisir là où le narrateur parle de remords, ce qui n'annule pas la signification immédiate et lui confère seulement une profondeur d'abord inaperçue.

Cette profondeur de l'écriture littéraire est aussi dépendante des procédés de fictionnalisation. Si à l'échelle macrotextuelle le livre de Rousseau ne se présente pas comme histoire inventée, donc comme roman, il raconte néanmoins toute une série d'anecdotes et de scènes qui ont fait les délices de la critique (Starobinski, 1970). Philippe 
Lejeune a montré de son côté comment l'histoire personnelle du petit Jean-Jacques est articulée par l'auteur au mythe de l'Âge d'or, reprenant à son compte la légende familiale. Rousseau s'y invente, à défaut de la mère qu'il n'a pu connaître, un père idéal, victime des méchants et qui a dû quitter la Cité contre son gré. Le mythe, version collective de la fiction, et la figure poétique, permettant de reconnaître par analogie un personnage sous un autre ${ }^{5}$, réintroduisent au sein d'un texte non fictionnel, une dimension fictive, propre à rassurer le narrateur scripteur, à masquer parfois des significations déplaisantes. Ils maintiennent de l'univoque là où le lecteur verra de l'équivoque.

Dans cette perspective, le recours au roman, forme plus ouvertement déclarée de fiction ${ }^{6}$, pourrait être la reconnaissance de la part de l'écrivain des limites de la démarche intentionnelle. S'abandonner à la forme romanesque, c'est s'exposer à l'équivoque interprétative et renoncer à la souveraineté sur ce qui se joue dans l'écriture.

L'Auteur en question

Cette posture, quand elle devient consciente, corrobore la critique radicale de la notion d'Auteur, menée à la fin des années 1960 sous l'impulsion de Barthes (1968) et de Foucault (1969). Ce dernier, notamment, insiste à juste titre sur l'historicité de la fonction d'auteur, inconnue au Moyen-Âge et qui se développe parallèlement à la propriété bourgeoise. Remettre ainsi l'auteur en perspective, c'est pour le moins relativiser sa prétention à une hégémonie sur le sens de ses écrits et légitimer par avance les équivoques reconnues par le seul lecteur. Faut-il pour autant substituer le lecteur à l'auteur au point de lui donner tous les pouvoirs d'interpréter ${ }^{7}$, comme semble y inviter la fin de l'article de Barthes et comme paraît encore le vouloir une part de la critique contemporaine confondant allègrement commentaire de texte et réécriture au premier degré ? Nous avons déjà critiqué cette attitude ${ }^{8}$ que Barthes corrigea dans ses écrits ultérieurs $(1970)^{9}$. II s'agit plutôt de tracer les contours d'un compromis entre les prérogatives de l'auteur (du texte) et celles du lecteur.

Protocole

Quoi qu'il en soit, la critique de l'intention littéraire amène à souligner les limites de la notion de pacte et peut-être à lui substituer celle de protocole. Le protocole tient compte de l'objet auquel il s'applique mais il ne requiert pas l'identification d'une intention. II est validé par la fécondité des résultats qu'il produit. La notion convient assez bien aux objets littéraires mal identifiés qui se multiplient à l'époque contemporaine. Prenons un exemple dans la

\footnotetext{
${ }^{5}$ Le fantasme de la marâtre, par exemple sous les traits de Mademoiselle Lambercier.

${ }^{6}$ Voir à ce sujet notre essai Le Roman de la lecture qui propose de distinguer trois degrés de la fiction : le roman comme fiction première, la figure poétique, comme fiction seconde et trace de l'imaginaire dans le discours, la fiction critique, enfin, comme fiction induite, tant il est vrai que le lecteur, si enclin soit-il à l'analyse rigoureuse et objective, ne peut faire abstraction de son imaginaire et de son inconscient.

7 "La naissance du lecteur doit se payer de la mort de l'Auteur », article cité, p. 45.

${ }^{8}$ Voir à ce sujet notre communication «Enjeux et limites de la création lectorale », Forum de l'APEF.

${ }^{9}$ Sade, Fourier, Loyola, par exemple, réintroduit à propos de l'auteur, la notion de biographème qui ouvre la voie à une récupération, au sein du processus interprétatif, d'éléments situés en amont du texte.
} 
nébuleuse improprement appelée autofictionnelle. Livret de famille de Patrick Modiano comporte quelques traits autobiographiques soulignés par le discours d'escorte éditorial. II y est question, comme dans de nombreux livres de l'auteur, de la guerre, de l'Occupation, d'un père cherché et dont l'identité et surtout le caractère semblent se dérober à l'enquête. Mais ce livret de famille, emblème de l'état civil et donc en principe de la quête autobiographique, comporte plus de faux noms que de vrais, plus de détails fictifs que d'éléments authentiques, certains étant de toute façon invérifiables sans consultation de sources extérieures. En tête du livre, aucune mention générique. La première des quatorze histoires ne propose pas de référence explicite à l'auteur, le je qui s'y exprime étant aussi bien celui qu'affectionnent certains romanciers. II faut attendre la seconde histoire, sans lien direct avec la première, pour voir surgir le nom de «P. Modiano ». Ecrire comme l'éditeur qu'il s'agit d'un livre « où l'autobiographie la plus précise se mêle aux souvenirs imaginaires ", apparaît donc abusif au regard d'une stratégie d'écriture fondée sur l'effacement des repères. L'autofiction appellerait à chercher Patrick Modiano à travers les différentes histoires, démarche sans doute possible en recoupant le livre avec d'autres traits de la vie de l'auteur collectés ici et là. Mais de toute évidence, l'entreprise scripturale de l'auteur ne correspond que très partiellement à ce projet et semble encore se chercher dans ses derniers écrits. II est en revanche possible au lecteur d'essayer divers protocoles de lecture, biographique, fantasmatique, sociohistorique, symbolique, susceptibles de donner sens à cette quête dont la portée dépasse l'individu Modiano. Univoque ou équivoque, l'interprétation lectorale sera à son tour jugée à l'aune des résultats produits et sans doute de leur capacité à donner chair à une lecture dans le respect du texte d'auteur.

Ayant montré comment, à propos de cette question de l'équivoque, il convient de dépasser la seule perspective auctoriale, il nous reste à examiner de plus près diverses modalités de l'interaction auteur/ lecteur.

\section{Le lecteur, l'équivoque littéraire et le partage des rôles}

Parce qu'elle articule des significations différentes, voire opposées, l'équivoque est à rapprocher du jeu, activité de synthèse qui met en relation des données de divers ordres avec des résultats plus ou moins harmonieux. Le jeu littéraire se joue avec un protagoniste principal, le texte à lire, d'où surgit une parole venue d'un autre. S'il veut tirer quelque profit de sa lecture, le lecteur a tout intérêt à prendre en compte la singularité de cette parole (Jenny, 1995). Il peut en résulter une coopération fructueuse ou d'autres formes plus conflictuelles. 


\section{Le jeu partagé, poésie, roman}

On a vu plus haut le cas d'une équivoque sonore involontaire. Certaines résultent sans doute de simples inadvertances et l'on connaît les plaisanteries de potaches sur deux ou trois vers isolés dans l'œuvre de Corneille. Ceci ne saurait faire oublier qu'une large part du plaisir poétique prend sa source dans l'équivoque sonore, reconnue et partagée. «II pleure dans mon cœur/ Comme il pleut sur la ville », écrit magnifiquement Verlaine. La trouvaille poétique réside dans la condensation en un seul énoncé de deux tournures verbales qui ne se contentent pas d'accorder la mélancolie intérieure et celle inspirée par le spectacle de la pluie ardennaise. Le néologisme grammatical «il pleure », calqué sur la tournure impersonnelle « il pleut » donne à éprouver l'état d'un sujet en proie, sous l'effet du malaise existentiel, à une dépossession de soi. Le plaisir poétique s'analyse en trois temps : un énoncé unique condense deux autres énoncés reconnus à l'oreille (équivoque sonore), la superposition des sens permet d'élaborer un troisième terme et de réduire l'équivoque en éprouvant par procuration (exorcisme poétique) la dépossession. D’autres effets sont bien sûr imaginables qui ne conduiraient pas à résoudre l'équivoque sonore en univocité sémantique.

Au plan romanesque, l'univocité du genre s'accommode d'équivoques de sens prises comme moyen de découverte de soi. L'espace fictionnel est en effet un espace intermédiaire réunissant sur le mode de la virtualité des éléments inventés et d'autres imités du monde réel. Ce qui s'y joue n'est pas réel mais modélise des expériences qui pourraient l'être. Le roman est aussi le lieu où la matière personnelle et biographique qui constitue le vécu auctorial subit une transmutation vers une vérité qui n'est plus tout à fait biographique ni personnelle. Dans W ou le souvenir d'enfance, Georges Perec entrelace subtilement autobiographie et fiction romanesque pour donner à éprouver cette transmutation. L'histoire personnelle est douloureuse, c'est celle de la déportation de sa famille, spécialement de sa mère dont il fut séparé en 1943. La fiction commence comme un roman d'aventure à la première personne par la quête d'un certain Gaspard Winckler parti à la recherche de son père et naufragé en mer avant qu'il ait pu atteindre l'île de W. Elle reprend dans la seconde moitié du livre avec la description à la troisième personne de la cité de $W$, modèle de société totalitaire utilisant le sport pour instrumentaliser les individus. Entre les deux parties, au milieu de son livre, Perec a placé le signe de l'ellipse « (...) ». La discordance des voix enchaînant au récit d'aventure la contre-utopie pose le problème de leur unification. La disparition du sujet narrant à la première personne introduit une distorsion à certains égards analogue à l'agrammaticalité verlainienne. La trouvaille esthétique réside dans l'exploitation de cette distorsion pour l'imaginaire, pour ce qu'elle invite à rétablir de liens entre la disparition d'un sujet et l'évocation poétique de l'indicible. La vie des êtres chers dans les camps reste en effet inaccessible au narrateur autobiographe. La société totalitaire de W en 
est la version allégorisée. Le roman serait ainsi, pour le lecteur comme pour l'auteur, participant du même jeu, expérience de l'indicible et reconstruction de soi par la traversée de l'innommable. II permettrait la réduction d'une forme extrême d'équivoque dans une unité supérieure.

\section{Discordance auteur/lecteur, rôle des intertextes}

Une telle harmonisation des rôles est toutefois loin d'être le cas de figure dominant. Rendue possible dans le cas du récit perecquien par la figure de l'ellipse qui offre aux deux parties un espace commun de non dit où se retrouver, elle ne saurait masquer la dissymétrie des positions du lecteur et de l'auteur, accentuée par le décalage temporel des expériences et la différence des horizons culturels.

Si l'on admet que de part et d'autre du texte se trouve sollicitée une activité inconsciente, il est possible d'imaginer une conjonction, comme on vient de le voir, mais aussi une disjonction. Tel est le cas des romans où domine l'affirmation de valeurs, on n'ose pas dire romans à thèses. Le cycle aragonien du Monde réel offre ici un exemple intéressant. Dans Les Voyageurs de l'impériale, l'auteur dépeint la décomposition de la société bourgeoise à la veille de la Seconde Guerre mondiale. Pierre Mercadier, le héros, quitte sa famille pour le mirage d'une autre vie qui l'emmènera notamment à Venise. Revenu à Paris, il meurt quasiment aphasique, capable d'articuler un seul mot qui résume ce qu'il a manqué dans sa vie : «Po-li-tique». Le foisonnement de l'écriture aragonienne ne réduit pas le message à construire à ce schéma univoque, mais la construction narrative lui confère une place de choix qui permit de l'inscrire dans le projet réaliste socialiste. II revient au lecteur de faire éclater cette univocité en s'appuyant sur ce que l'écriture donne aussi à entendre sur un mode mineur. Les intertextes jouent ici un rôle spécial : l'imaginaire aragonien apparaît toujours hanté par le souvenir fort peu édifiant des Chants de Maldoror. On retrouve même dans une des strophes de Lautréamont une histoire avec bus à impériale et quelques autres éléments communs, ce qui autorise à formuler l'hypothèse d'une présence latente de cet intertexte dans Les Voyageurs (Trouvé, 2004). De même le récit des Communistes qui s'apparente parfois à la Geste du Parti offre à qui veut bien le lire attentivement nombre de décrochements vers une esthétique de la discordance. Je pense ici à la vision baroque et hallucinatoire du désastre de Dunkerque qui renvoie à la peinture de Bruegel (Vigier, 2000). Susan Suleiman a montré dans son essai sur Le roman à thèse (1983) les failles introduites dans le système axiologique dominant par l'écriture littéraire. Ceci vaut d'autant plus que l'écrivain est de qualité. II n'empêche : la restitution de l'équivoque s'opère ici avec et contre ce que le texte donne à lire de façon immédiate.

\section{Trouble identitaire partagé}

Auteur et lecteur peuvent enfin se rejoindre dans l'orchestration et la perception d'un trouble identitaire qui serait encore une équivoque de sens, la plus profonde, peut-être. Le 
roman est ici une forme privilégiée. L'œuvre de Modiano déjà évoquée pourrait se lire en termes de construction/ déconstruction des certitudes identitaires. II est frappant à ce sujet d'observer l'effacement progressif des repères intertextuels offerts au lecteur comme autant de signes de reconnaissance. Au feu d'artifice intertextuel de La Place de l'étoile, roman princeps qui signe l'entrée fracassante de l'auteur dans le monde des lettres, succède une écriture du dépouillement qui s'emploie à miner les certitudes. Rue des boutiques obscures constitue à cet égard un moment fort. La fausse enquête policière mène le lecteur à des révélations en trompe-l'œil. Au passage on croit pouvoir reconnaître des allusions intertextuelles. Le directeur de l'Agence qui a embauché comme détective privé le narrateur amnésique fait ses bagages pour le Midi. Le souvenir d'Anouilh (Le voyageur sans bagage) s'invite dans la lecture. Le narrateur déambule dans des lieux supposés recéler des traces de son passé qui ranimeraient sa mémoire ; l'impression de déjà vu attachée à certaines descriptions fait penser à Nerval sans qu'une certitude puisse être établie. C'est tout un arrière-plan intertextuel ou arrière-texte ${ }^{10}$ qui nourrit ici la catégorie de l'équivoque.

D'une autre façon, le livre de la hongroise Magda Szabó L'Instant La Créüside illustre les potentialités équivoques du genre romanesque. Dans cette réécriture parodique et féministe de L'Enéide, l'auteur imagine un autre destin pour Créüse, l'épouse du Bon Père, fondateur de Rome, Créüse, que le récit épique fait disparaître dans les flammes de Troie, au moment de la fuite du héros portant, selon la légende, Anchise sur ses épaules. Lors de l'instant où tout bascule, Szabó imagine que c'est Enée qui a été tué, Créüse accomplissant à sa place le périple vers Carthage puis l'Italie, avant de revenir - réécriture oblige - sur le sol troyen. Pour les compagnons du héros, il est entendu qu'Enée est toujours à leur tête, Enée qui a pris les traits de Créüse sous l'effet d'un sortilège divin. Plusieurs chapitres placent le lecteur devant la troublante question de l'identité sexuelle : Enée parle et ce n'est pas lui. Le roman pousse à son paroxysme ce qui est peut-être l'essence de l'interrogation romanesque : l'identité de genre et de sexe (Derrida, 1994) ${ }^{11}$, question hors du propos de l'épopée. L'œuvre revêt aussi, dans le contexte des années 1980, une connotation antistalinienne et souligne indirectement ce que l'épopée virgilienne représente d'allégeance à un pouvoir impérial totalitaire. L'étonnant Avant-propos dévoile les dessous de l'histoire par la parole d'un auteur lui-même dédoublé : le poète Saboas explique que faire mourir l'épouse d'Enée était le prix à payer pour justifier l'exil du bon père et la fondation sur le sol italien d'une lignée d'origine divine à la gloire de l'empereur Auguste, la poétesse Szabó conte la genèse elle-même romanesque de son histoire à la veille de la chute du Mur.

\footnotetext{
10 «Intertexte et arrière-texte » est le thème du séminaire rémois Approches Interdisciplinaires de la Lecture, 2009-2010 (dir. Marie-Madeleine Gladieu \& Alain Trouvé), actes à paraître ultérieurement.

11 Jacques Derrida écrit à ce propos: «Tout récit fabuleux raconte, met en scène, enseigne ou donne à interpréter la différence sexuelle » («Fourmis », in Lectures de la différence sexuelle).
} 
De multiples façons, la question de l'équivoque apparaît donc comme une question de lecture : le décodage de repères génériques plus ou moins clairs s'analyse en termes de pacte littéraire ; l'exploitation de potentialités textuelles échappant à l'intention auctoriale invite à trouver un protocole de lecture adéquat qui fasse résonner l'œuvre sans la trahir ; l'entreprise interprétative s'empare de l'équivoque, cherchant à la réduire ou à la cultiver.

L'équivoque qui s'appréhende au niveau du genre, donc d'une première lecture cursive, se retrouve au coeur de l'interprétation, lorsque s'élabore le sens du texte lu. Aux deux niveaux, elle se nourrit de l'univoque, dont elle se distingue, et tend à le recréer sous la forme d'une unité supérieure.

La fiction (spécialement le roman) entretient un rapport privilégié avec l'équivoque. La récusation de l'équivoque générique (il faut que la fiction décolle du récit factuel) semble donner son plein essor à l'exploration des équivoques identitaires qui en constitue le fonds.

Comme l'écrivain mais de façon décalée, tantôt concordante, tantôt dissonante, le lecteur semble jouer à résorber et à faire renaître l'équivoque, dont le vocable s'autodécrit, renvoyant à la coexistence de sens différents voire opposés ou à l'équivalence des contraires.

Par tous ces aspects, le jeu avec l'équivoque occupe une place cruciale au sein de l'activité littéraire. 


\section{Bibliographie}

BARTHES, Roland $(1968,2002)$. «La Mort de l'Auteur », Manteia, n5. In: CEuvres Complètes, III, pp. 40-45.

BARTHES, Roland (1970). Sade, Fourier, Loyola. Paris: Le Seuil.

DERRIDA, Jacques (1994). "Fourmis ». In: Lectures de la différence sexuelle, textes réunis et présentés par Mara Negron. Paris: Des femmes.

FOUCAULT, Michel (1969, 1994). "Qu'est-ce qu'un auteur? ", Bulletin de la société française de philosophie, $63^{\text {ème }}$ année, ${ }^{\circ} 3$. In: Dits et Ecrits, I. Paris: Gallimard.

JENNY, Laurent (1995). La Parole singulière. Paris: Belin.

LEJEUNE, Philippe (1975). Le Pacte autobiographique. Paris: le Seuil.

LUKÀCS, Georg (1916, 1979). La Théorie du roman. Paris: Denoël-Gonthier, pp. 49 à 60.

MARTIN, Joël (2009). La contrepèterie. Paris: PUF, « Que sais-je ? », n³740.

RANCIÈRE, Jacques (2007). Politique de la littérature. Paris: Galilée.

RANCIÈRE, Jacques (2008). Le Spectateur émancipé. Paris: Galilée.

RICOEUR, Paul (1990). Soi-même comme un autre. Paris: le Seuil.

STAROBINSKI, Jean $(1970,2001)$. «Le dîner de Turin ». In: La Relation Critique. Paris: Gallimard, pp. 126-186.

STAROBINSKI, Jean (1971). Jean-Jacques Rousseau La transparence et l'obstacle. Paris: Gallimard. SULEIMAN, Susan (1963). Le roman à thèse ou l'autorité fictive. Paris: PUF.

TROUVÉ, Alain (2004). Le Roman de la lecture. Liège: Mardaga.

TROUVÉ, Alain (2004). «Mystification et jeu littéraire ». In: Mélire ? Mystification et jeu littéraire, dir. Nathalie Preiss. Paris: L'Improviste, pp. 135-147.

TROUVÉ, Alain (2008). «Enjeux et limites de la création lectorale ». In: Forum de l'APEF, « Cultures littéraires : nouvelles performances et développement ».

VIGIER, Luc (2000). "Dunkerque ou le témoignage lacéré ». In: Lire Aragon (dir. M. Hilsum, C. Trévisan, M. Vassevière). Paris: Champion, pp. 263-278. 